LPT-ORSAY 00/70

UHU-FT/00-02

\title{
Consistent OPE Description of Gluon Two- and Three-point Green Functions?
}

\author{
Ph. Boucaud ${ }^{a}$, A. Le Yaouanc ${ }^{a}$, J.P. Leroy ${ }^{a}$, J. Micheli ${ }^{a}$, \\ O. Pène ${ }^{a}$, J. Rodríguez-Quintero ${ }^{b}$ \\ ${ }^{a}$ Laboratoire de Physique Théorique円 \\ Université de Paris XI, Bâtiment 210, 91405 Orsay Cedex, France \\ ${ }^{b}$ Dpto. de Física Aplicada e Ingeniería eléctrica \\ E.P.S. La Rábida, Universidad de Huelva, 21819 Palos de la fra., Spain
}

\begin{abstract}
We perform an OPE analysis of the flavorless non-perturbative gluon propagator and the symmetric three-gluon vertex in the Landau gauge. The first subdominant operator is $A_{\mu} A^{\mu}$ which can condensate in the Landau gauge "vacuum" although being a non-gauge invariant operator. We neglect all higher dimension operators. Then the gluon propagator and the symmetric three gluon vertex only depend on one common unknown condensate. We propose a consistency check from lattice data. At two loops for the leading coefficient and with $1 / p^{2}$ corrections at tree-level order the two fitted values for the condensate do not agree. At three loops we argue that the today unknown $\beta_{2}^{\mathrm{MOM}}$ should be equal to $1.5(3) \times \beta_{2}^{\mathrm{MOM}}=7400(1500)$ to fulfill the OPE relation. Inclusion of the power corrections' anomalous dimensions should improve further the agreement. We show that these techniques cannot be applied to the asymmetric three gluon vertex with one vanishing momentum.
\end{abstract}

P.A.C.S.: $12.38 . \mathrm{Aw} ; 12.38$.Gc; $12.38 . \mathrm{Cy} ; 11.15 . \mathrm{H}$

\section{Introduction}

Much effort has being devoted in the last years to the non-perturbative computation of the running QCD coupling constant $\alpha_{s}(p)$ [1] [7]. A most surprising result arising from the lattice computation of the gluon three-point Green function [7] is that a $\sim 1 / p^{2}$ power correction is still sizeable for momenta of the order of $\sim 10 \mathrm{GeV}$ !!

These power corrections were qualitatively discussed in [7] in connection with several physics phenomena such as interquark potential, the gluon condensate puzzle, Landau pole, etc... It was also strongly stressed that $\alpha_{s}(p)$ being non-perturbatively defined on a lattice in the particular Landau gauge, a contribution to the operator product expansion (OPE) from a gauge dependent local operator should not a priori be neglected. More precisely, the $A^{2}=A_{\mu} A^{\mu}$ operator can acquire a vacuum expectation value (v.e.v.) because of the non-gauge-invariant nature of the vacuum. As a matter of fact, the authors of ref. [9] showed in a general way that the OPE for QCD propagators unequivocally generates non null v.e.v. of $A_{\mu} A^{\mu}$. The important point is that in contrast to that of the gauge invariant $G^{\mu \nu} G_{\mu \nu}$ generating $1 / p^{4}$ powers, it produces $1 / p^{2}$

\footnotetext{
${ }^{1}$ Unité Mixte de Recherche du CNRS - UMR 8627
} 
corrections which may be of comparable size to higher perturbative correction (three-loops, for instance) for the considered momenta.

The key remark here is that $A^{2}$ is the only dimension-two operator able to generate a non vanishing v.e.v. The purpose of the present paper is to exploit this fact according to OPE philosophy in the following manner: we will study both the gluon propagator and the three gluon vertex (two- and three-point Green functions) in order to examine whether the same $\left\langle A^{2}\right\rangle$ condensate can consistently describe the evaluated power corrections to both quantities. For rather obvious reasons which will be explicited later, we will totally neglect $O\left(1 / p^{4}\right)$ corrections.

The OPE approach consists in expanding the Green functions in inverse power series in $p^{2}$, every term corresponding to a local operator. The coefficients which multiply the operators are calculable in perturbation 10. In practice of course the coefficients are expanded to a given finite order, not only because the perturbative series are only asymptotic, but also because computing higher orders is a rapidly increasing task which has not yet been performed.

The potential dangers when using OPE to relate different Green functions via the same condensates has been stressed by several authors [11, 12]: it was claimed that the coefficients of the leading operator at any given momentum scale have to be computed to a sufficiently high perturbative order to make the perturbative uncertainty smaller than the power corrections.

Our input data are lattice computed Green functions [5], covering a momentum range from 2 to $10 \mathrm{GeV}$. We will analyze them according to OPE. The gluon propagator and the symmetric three-point Green function $\left(p_{1}^{2}=p_{2}^{2}=p_{3}^{2}\right)$ can be expanded into perturbatively computable coefficients and the only unknown $\left\langle A^{2}\right\rangle$ condensate. This allows to perform a test of OPE in a relatively favorable situation. Our first "ace" is indeed this wide momentum window which reduces the risk of confusion between neglected perturbative (logarithmic) contributions and the $\sim 1 / p^{2}$ terms.2. Our second "ace" is that $1 / p^{2}$ terms are rather easy to display in the $2-10$ $\mathrm{GeV}$ window [7] while, for example, $1 / p^{4}$ contributions would only influence the lower part of this window making the fits hazardous. Our third "ace" is the high accuracy achieved by our lattice calculations.

The leading coefficient (the coefficient of identity operator in the OPE) of the gluon propagator is known up to three loops but the symmetric three-gluon vertex is only known at two loops. To ask for an OPE consistent description when leading power coefficients are only expanded at two loops might be exposed to the danger mentioned above [12]. We intend to check this. We will also try the three-loop consistent description by fitting the unknown perturbative information for the three-gluon vertex, the $\beta_{2}$ parameter of beta function in MOM scheme. The comparison with future perturbative computations will obviously be in order.

We have only computed at tree-level order the perturbative coefficients multiplying the $A^{2}$ operator. We will therefore perform the fits with a constant times $1 / p^{2}$ term. A knowledge of the anomalous dimension of the coefficient which multiplies $1 / p^{2}$ will be welcome [15].

The theoretical setting of our use of OPE is described in Section 2. A preliminary analysis of results from previous lattice data are presented in Section 3 . We finally discuss and conclude in Section 4 .

\footnotetext{
${ }^{2}$ Still it should be noted that the recent perturbative computation of $\beta_{3}$ in asymmetric MOM scheme 14 leads to a four-loop estimate of the empiric power coefficient introduced in ref. [f] wich is roughly 0.8 times the threeloop one. This shows that some amount of disguise of higher order perturbative terms into power corrections is difficult to fully avoid.
} 


\section{OPE for the gluon propagator and $\alpha_{s}(p)$}

The purpose of the present section is to develop an OPE-based model describing the nonperturbative power behaviour of gluon propagator and $\alpha_{s}(p)$. To this goal, we will compute the first power correction in OPE (always at tree-level) for two and three gluon operators, by following the prescriptions of [10]. Some general results for the gluon propagator in full QCD can be found in [9]. For completeness we will describe our own calculations for both the propagator and the three gluon vertex. We have checked agreement for the propagator with [9].

The two- and three-point bare lattice Green functions are renormalized by imposing momentum subtraction prescriptions. Let us insist: such a renormalization prescriptions leads to the definition of a gauge-dependent coupling which consequently admits a non vanishing contribution from the non-gauge invariant $\mathrm{OPE} A_{\mu} A^{\mu}$.

We work in the pure Yang-Mills QCD, without quarks. OPE yields

$$
\begin{aligned}
T\left(\widetilde{A}_{\mu}^{a}(-p) \widetilde{A}_{\nu}^{b}(p)\right) & = \\
\left(c_{0}\right)_{\mu \nu}^{a b}(p) 1+ & \left(c_{1}\right)_{\mu \nu a^{\prime}}^{a b \mu^{\prime}}(p): A_{\mu^{\prime}}^{a^{\prime}}(0):+\left(c_{2}\right)_{\mu \nu a^{\prime} b^{\prime}}^{a b \mu^{\prime} \nu^{\prime}}(p): A_{\mu^{\prime}}^{a^{\prime}}(0) A_{\nu^{\prime}}^{b^{\prime}}(0): \\
& +\ldots, \\
T\left(\widetilde{A}_{\mu}^{a}\left(p_{1}\right) \widetilde{A}_{\nu}^{b}\left(p_{2}\right) \widetilde{A}_{\rho}^{c}\left(p_{3}\right)\right) & = \\
\left(d_{0}\right)_{\mu \nu \rho}^{a b c}\left(p_{1}, p_{2}, p_{3}\right) 1 & +\left(d_{1}\right)_{\mu \nu \rho a^{\prime}}^{a b c \mu^{\prime}}\left(p_{1}, p_{2}, p_{3}\right): A_{\mu^{\prime}}^{a^{\prime}}(0): \\
& +\left(d_{2}\right)_{\mu \nu \rho \mu^{\prime} b^{\prime}}^{a b c \mu^{\prime}}\left(p_{1}, p_{2}, p_{3}\right): A_{\mu^{\prime}}^{a^{\prime}}(0) A_{\nu^{\prime}}^{b^{\prime}}(0):+\ldots ;
\end{aligned}
$$

written only in terms of normal products of local gluon field operators, where $A(\widetilde{A})$ stands for the gluon field in configuration (momentum) space, $a, b$ being colour indices and $\mu, \nu$ Lorentz ones. The notation $T()$ simply refers to the standard $T^{*}$ product in momentum space. The normal product of Eqs. (1, 2, will be defined below in reference to the perturbative vacuum. The r.h.s. in eqs. (1, 2, contains operators of dimension up to two. We have omitted local operators including, for instance, gluon field derivatives or ghosts. Indeed at the order $1 / p^{2}$, which we consider in this letter, they have a vanishing v.e.v. (Obviously $<\partial_{\mu} A^{\mu}>=0$ ).

\section{$2.1 \quad$ Gluon propagator}

The following step is to take the QCD v.e.v. (in our flavorless universe) in both r.h.s. and l.h.s. of equation (1),

$$
\left\langle T\left(\widetilde{A}_{\mu}^{a}(-p) \widetilde{A}_{\nu}^{b}(p)\right)\right\rangle=\left(c_{0}\right)_{\mu \nu}^{a b}\left(p^{2}\right)+\left(c_{2}\right)_{\mu \nu a^{\prime} b^{\prime}}^{a b \mu^{\prime} \nu^{\prime}} \delta^{a^{\prime} b^{\prime}} g_{\mu^{\prime} \nu^{\prime}} \frac{\left\langle A^{2}\right\rangle}{4\left(N_{c}^{2}-1\right)}+\ldots
$$

where $A^{2}=: A_{\mu^{\prime}}^{a^{\prime}}(0) A_{a^{\prime}}^{\mu^{\prime}}(0):$. A first immediate simplification in r.h.s. of Eq. (3) with regard to Eq. (1) is the disappearance of terms containing an odd number of local gluon field operators. They contribute only at higher orders in the OPE because of the impossibility to build a Lorentz invariant tensor (with odd number of indices) without any external non-null 
momentum. The normal product in eqs. (10,2) refers to the perturbative vacuum $\mid 0>$. To be more specific the non-perturbative condensate $\left\langle A^{2}\right\rangle$ is defined from eq. (3) as follows : given the l.h.s computed non-perturbatively on a lattice ${ }^{m}$, given the coefficients $c_{0}$ and $c_{2}$ in the r.h.s computed perturbatively at a given order in perturbation theory and for a particular renormalization momentum scale and scheme $\left\langle A^{2}\right\rangle$ is unambiguously defined.

The standard method 10 to compute the perturbative expansion of the OPE Wilson coefficient is to compute to the wanted order an appropriate matrix element of the 1.h.s. operator of Eqs. (1,2). The perturbative gluon propagator

$$
\left(G_{p e r t}^{(2)}\right)_{\mu \nu}^{a b}\left(p^{2}\right)=\left\langle 0\left|T\left(\widetilde{A}_{\mu}^{a}(-p) \widetilde{A}_{\nu}^{b}(p)\right)\right| 0\right\rangle
$$

at the considered order in perturbation theory yields $c_{0}$ while $c_{2}$ may be obtained from

$$
\left\langle g\left|T\left(\widetilde{A}_{\mu}^{a}(-p) \widetilde{A}_{\nu}^{b}(p)\right)\right| g\right\rangle_{\text {connected }}=\left(c_{2}\right)_{\mu \nu a^{\prime} b^{\prime}}^{a b \mu^{\prime} \nu^{\prime}}(p)\left\langle g\left|: A_{\mu^{\prime}}^{a^{\prime}}(0) A_{\nu^{\prime}}^{b^{\prime}}(0):\right| g\right\rangle
$$

with two soft external gluons. From

$$
\left\langle g\left|A^{2}\right| g\right\rangle=2+O(\alpha)
$$

we may compute $c_{2}$. At tree-level order the $O(\alpha)$ is neglected and eq. (5) allowing to compute $c_{2}$ from the computation of the l.h.s. : f

$$
\left(c_{2}\right)_{\mu \nu a^{\prime} b^{\prime}}^{a b \nu^{\prime} \nu^{\prime}}=\frac{1}{2} \frac{\left\langle\widetilde{A}_{\tau}^{t}(0) \widetilde{A}_{\mu}^{a}(-p) \widetilde{A}_{\nu}^{b}(p) \widetilde{A}_{\sigma}^{s}(0)\right\rangle}{\left(G_{\text {pert }}^{(2)}(0)\right)_{\tau \mu^{\prime}}^{t a^{\prime}}\left(G_{\text {pert }}^{(2)}(0)\right)_{\sigma \nu^{\prime}}^{s b^{\prime}}} .
$$

The ratio in the r.h.s of Eq. (77) represents symbolically diagrams with four gluon legs where the two of them carrying zero momentum are explicitly cut. Consistently with Eq. (6), we compute in Landau gauge the tree-level diagrams shown in fig. 1 to obtain $\Gamma_{\mu \nu t^{\prime} s^{\prime}}^{a b \sigma^{\prime}}$ and then, for $p^{2}=-k^{2}$, we can write:

$$
\begin{array}{r}
\left\langle T\left(\widetilde{A}_{\mu}^{a}(-p) \widetilde{A}_{\nu}^{b}(p)\right)\right\rangle=\frac{-i}{-k^{2}}\left(g_{\mu \nu}-\frac{p_{\mu} p_{\nu}}{-k^{2}}\right) \delta^{a b} \\
\times\left(-k^{2} G_{(\mathrm{n} \text { loops })}^{(2)}\left(k^{2}\right)+\frac{3 g^{2}\left\langle A^{2}\right\rangle}{4\left(N_{c}^{2}-1\right)} \frac{1}{-k^{2}}+O\left(\alpha^{n+1}, \alpha^{2} \frac{\Lambda^{2}}{-k^{2}}\right)\right),
\end{array}
$$

As already mentioned, there is a series of problems in the practical use of OPE tried in this paper [11, 12]. In general, the whole expansion in terms of condensates suffers from the socalled renormalon ambiguities : the resummation of the non-Borel-summable perturbative series for the leading operator Wilson coefficient is ambiguous, this ambiguity being compensated by the ultraviolet renormalon ambiguity of the matrix element of the subdominant operator $A^{2}$. By luck the infrared renormalon ambiguity cancels off when comparing the contribution of the condensate $<A^{2}>$ in both the propagator and the three gluon vertex. In this way we may

\footnotetext{
${ }^{3}$ We thank Y. Dokshitzer and G. Korchemsky for illuminating discussions on this point.

${ }^{4}$ Being a gauge dependent quantity it cannot be accessed from experiment.

${ }^{5}$ Beyond tree-level order the radiative corrections, $O(\alpha)$ terms in (6), are calculable, but we will skip this issue in this paper.
} 


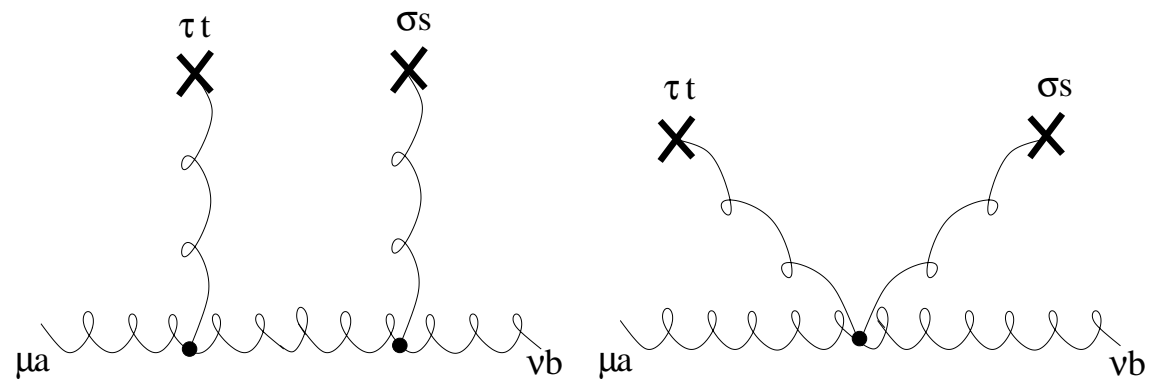

Figure 1: Four gluons tree-level diagrams contributing (with all their possible permutations) to the $\left(c_{2}\right)_{\mu \nu t s}^{a b \tau \sigma}$ OPE perturbative coefficient of the gluon propagator. Crosses mark the gluon legs due to the external soft gluons.

simply decide to truncate the perturbative series to some order and the universality of the condensates is preserved provided this order is clearly stated.

A remnant of this renormalon problem arises from the truncation of OPE and perturbative series for the Wilson coefficients. One has to be sure to have gone far enough in perturbative expansions and in the inverse power expansion to have a coherent scheme. We will try to justify later our truncation of the power series to order $1 / k^{2}$. For the perturbative series of the leading contribution we have to choose a truncation order $n$ according to a compromise: it should be high enough to make sure of the dominance of non-perturbative contributions compared to the perturbative uncertainty from neglected higher orders; but low enough not to destroy the good asymptotic behaviour of the series.

The gluon propagator turns out to be known up to the third loop in its perturbative expansion [6, 13], but not yet the symmetric MOM coupling constant. We can thus try the exercise with both $n=2$ and $n=3$, but making in the latter case some fit for the unknown three loop coefficient $\left(\beta_{2}\right)$. This is not too high an order over the considered momentum window but it might be too low. Concerning power corrections higher than $1 / k^{2}$ : our fits do not show the necessity for $1 / k^{4}$ terms (which would exhibit a very distinguishable functional dependence) over the large momenta window we consider. This is lucky since in practice it would be hopeless to deal with $1 / k^{4}$ terms, too many operators being needed as well as, for consistency, many higher orders in the perturbative expansion of the $c_{0}, d_{0}, c_{2}$ and $d_{2}$ coefficients. Anyhow the consistency of the matching to lattice non-perturbative data for the two- and three-point Green functions will allow to test the validity of our assumptions.

In brief, within the scope of the present paper we will satisfy ourselves by modeling power contributions to our Euclidean two-point Green function,

$$
\left\langle\widetilde{A}_{\mu}^{a}(-p) \widetilde{A}_{\nu}^{b}(p)\right\rangle=\delta_{a b}\left(\delta_{\mu \nu}-\frac{p_{\mu} p_{\nu}}{p^{2}}\right) G^{(2)}\left(p^{2}\right)
$$

such that

\footnotetext{
${ }^{6}$ In the $\widehat{\mathrm{MOM}}$ scheme the fourth loop coefficient of the $\beta$ function is known 14 and the series looks like being in its perturbative regime for momenta higher than $\sim 3 \mathrm{GeV}$
} 


$$
k^{2} G^{(2)}\left(k^{2}\right)=Z^{M O M}\left(k^{2}\right)=Z_{\text {nloops }}^{M O M}\left(k^{2}\right)+\frac{3 g^{2}\left\langle A^{2}\right\rangle}{4\left(N_{c}^{2}-1\right)} \frac{1}{k^{2}},
$$

where $Z_{\text {nloops }}^{M O M}=k^{2} G_{\text {nloops }}^{(2)}, n=2,3$ and by studying systematically the stability of the matching of lattice results to formula (10) for different choices of the momentum window.

All the quantities in Eqs (3,10) are bare objects, where the explicit dependence on the regularization cut-off has been omitted for the sake of simplicity. However, applying MOM prescription at a certain renormalization scale $\mu^{2}$, a renormalized version of Eq. (10) is obtained,

$$
k^{2} G_{R}^{(2)}\left(k^{2}, \mu^{2}\right)=Z_{\mathrm{R}, \text { nloops }}^{\text {MOM }}\left(k^{2}, \mu^{2}\right)+c\left(k^{2}, \mu^{2}\right) \frac{\left\langle A^{2}\right\rangle_{R, \mu^{2}}}{4\left(N_{c}^{2}-1\right)},
$$

where, in MOM philosophy, $\left\langle A^{2}\right\rangle_{R, \mu^{2}}$ is renormalized at $\mu^{2}$ such that the Wilson coefficient takes the tree-level value computed from the diagrams of fig. 1 : $c\left(\mu^{2}, \mu^{2}\right)=3\left(g_{R}\left(\mu^{2}\right)\right)^{2} / \mu^{2}$. $Z_{\mathrm{R}, \text { nloops }}^{M O M}\left(k^{2}, \mu^{2}\right)$ in Eq. (11) behaves according to the three-loop (two-loop) anomalous dimension of the gluon propagator [6] and is such that $\mu^{2} G_{R}^{(2)}\left(\mu^{2}, \mu^{2}\right)=1$. This last condition imposes an overall normalization which is totally irrelevant for our lattice data fitting strategy. In this respect the situation will slightly differ in the next section. By definition the running $k^{2}$ is there the renormalization point for the running coupling constant. It is then the condensate renormalized at $k^{2},\left\langle A^{2}\right\rangle_{R, k^{2}}$, and the coefficient accordingly renormalised at $k^{2}$ which have to be dealt with. This has to be taken into account when computing the anomalous dimension of the factor multiplying $1 / k^{2}$ in each case as will be done soon [15]. Nevertheless, the goal of the present paper is to develop the principle of an OPE testing procedure and to perform a preliminary check. In the scope of this preliminary check, where only tree-level power corrections are taken into account, we will simply take zero anomalous dimensions :

$$
c\left(k^{2}, \mu^{2}\right)\left\langle A^{2}\right\rangle_{R, \mu^{2}}=3 g_{R}^{2} \frac{\left\langle A^{2}\right\rangle_{R}}{k^{2}},
$$

where $g_{R}^{2}\left\langle A^{2}\right\rangle_{R}$ is taken to be a non-perturbative constant which can be fitted from lattice data.

\subsection{Running coupling constant}

In momentum subtraction schemes, in Landau gauge, the renormalized coupling constant can be defined at the renormalization momentum scale $k^{2}$ as (See for instance [5]) :

$$
g\left(k^{2}\right)=\frac{G^{(3)}\left(p_{1}^{2}, p_{2}^{2}, p_{3}^{2}\right)\left(Z^{M O M}\left(k^{2}\right)\right)^{3 / 2}}{G^{(2)}\left(p_{1}^{2}\right) G^{(2)}\left(p_{2}^{2}\right) G^{(2)}\left(p_{3}^{2}\right)},
$$

where $Z^{M O M}$ and $G^{(2)}$ were defined in the previous subsection, and $G^{(3)}\left(p_{1}^{2}, p_{2}^{2}, p_{3}^{2}\right)$ is the threepoint scalar form factor. Eq. (13) is directly written for an Euclidean metric. Nevertheless, as done in the previous section, we prefer to compute the scalar form factor in the more familiar Minkowskian metrics and translate the final result to Euclidean. This form factor should 
be obtained by projecting of the three-point Green function, $\left\langle\widetilde{A}_{\mu}^{a}\left(p_{1}\right) \widetilde{A}_{\nu}^{b}\left(p_{2}\right) \widetilde{A}_{\rho}^{c}\left(p_{3}\right)\right\rangle$, over the transverse tree-level three-gluon tensor,

$$
\left(T^{\text {tree }}\right)_{\mu_{1} \mu_{2} \mu_{3}}=\left[g^{\mu_{1}^{\prime} \mu_{2}^{\prime}}\left(p_{1}-p_{2}\right)^{\mu_{3}^{\prime}}+\text { cycl.perm. }\right] \prod_{i=1,3}\left(g_{\mu_{i}^{\prime} \mu_{i}}-\frac{p_{i \mu_{i}^{\prime}} p_{i \mu_{i}}}{p_{i}^{2}}\right)
$$

considered for a given configuration of the three momenta with an overall renormalization scale $k^{2}$. In the following we shall consider the two standard momentum subtraction kinematics: $p_{1}^{2}=p_{2}^{2}=p_{3}^{2}=-k^{2}(\mathrm{MOM})$ and $p_{1}^{2}=p_{3}^{2}=-k^{2}, p_{2}^{2}=0(\widetilde{\mathrm{MOM}})$.

In the MOM case, the scalar form factor, $G^{(3)}\left(k^{2}, k^{2}, k^{2}\right)$, is obtained from:

$$
\begin{aligned}
& G^{(3)}\left(k^{2}, k^{2}, k^{2}\right) f^{a b c}=\frac{1}{-18 k^{2}}\left\langle\widetilde{A}_{\mu}^{a}\left(p_{1}\right) \widetilde{A}_{\nu}^{b}\left(p_{2}\right) \widetilde{A}_{\rho}^{c}\left(p_{3}\right)\right\rangle \\
& \times\left[\left(T^{\text {tree }}\right)^{\mu_{1} \mu_{2} \mu_{3}}+\frac{\left(p_{1}-p_{2}\right)^{\rho}\left(p_{2}-p_{3}\right)^{\mu}\left(p_{3}-p_{1}\right)^{\nu}}{-2 k^{2}}\right] .
\end{aligned}
$$

Then, we follow from Eq. (2) a procedure to obtain the OPE perturbative coefficient $d_{2}$ analogous to that to the one previously used for the gluon propagator. The diagrams to compute are those plotted in Fig. 2. In total analogy to the gluon propagator case, the result for the tree-level OPE correction of the three-point Green function, translated to Euclidean metrics, leads to

$$
G^{(3)}\left(k^{2}, k^{2}, k^{2}\right)=G_{\text {pert }}^{(3)}\left(k^{2}, k^{2}, k^{2}\right)+\frac{9 g^{3}\left\langle A^{2}\right\rangle}{4\left(N_{c}^{2}-1\right)} \frac{1}{k^{2}} ;
$$

where, again, $G_{\text {pert }}^{(3)}$ stands for the purely perturbative three-point form factor.

Then, we insert Eqs. (10,16) in the MOM coupling constant definition, Eq. (13), where the Green functions scalar form factors are automatically renormalized in MOM prescription at the scale $k^{2}$ by the $3 / 2$-power of $Z^{M O M}$, and finally obtain

$$
\alpha_{s}{ }^{\operatorname{MOM}}\left(k^{2}\right)=\left(\alpha_{s}{ }^{\operatorname{MOM}}\left(k^{2}\right)\right)_{\text {pert }}\left[1+\frac{g_{R}^{2}\left\langle A^{2}\right\rangle_{R}}{4\left(N_{c}^{2}-1\right)} \frac{1}{k^{2}}(9+O(\alpha))\right] .
$$

One interesting remark is that all the OPE contribution to $\alpha_{s}{ }^{\mathrm{MOM}}\left(k^{2}\right)$ defined by Eq. (13) is coming from the explicit renormalization factor $\left(Z^{M O M}\right)^{3 / 2}$. After amputation of the external legs, diagrams (a) and (b) in fig 2 are obviously canceled, and a direct cancellation of the irreducible diagrams (c) and (d) happens.

As already mentioned only the two-loop coefficient of the beta function is known in the symmetric MOM scheme. The gluon propagator anomalous dimension is known at three loops

\footnotetext{
${ }^{7}$ The $O(\alpha)$-corrections in the second r.h.s. of Eq. (17) are of the order of the assumed theoretical uncertainty in our tree-level OPE correction.

${ }^{8}$ the two first coefficients, $\beta_{0}$ and $\beta_{1}$ are universal
} 

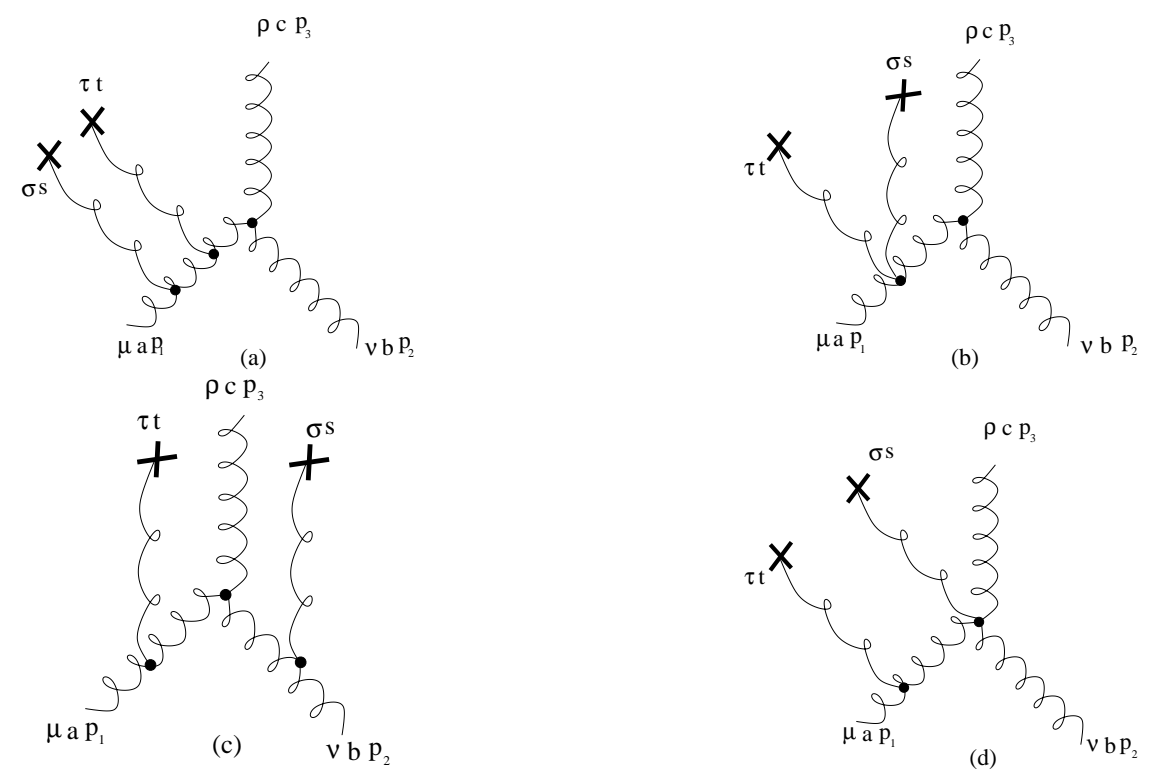

Figure 2: Five gluons diagrams to be computed, among all the possible new ones obtained by permutations of leg labels, in order to determine the tree-level OPE correction to the gluon three-point Green function.

and previous works [6] has revealed the important role played by the third loop in its appropriate perturbative description. Moreover, a previous analysis [7] including power corrections of $\alpha_{s} \widehat{\mathrm{MOM}}(k)$, in $\widehat{\mathrm{MOM}}$ scheme, also stressed the necessity of working at least at three-loops to get a good estimate of $\Lambda_{\overline{\mathrm{MS}}}$. This is why we shall add to the two loop test of OPE a three-loop one with a fitted $\beta_{2}$. The latter test is performed consistently at the three-loop level both for the gluon propagator renormalization constant, $Z^{M O M}\left(k^{2}\right)$, and for the perturbative part of $\alpha_{s}{ }^{\operatorname{MOM}}\left(k^{2}\right)$, which we will take to be the numerical inversion of

$$
\begin{aligned}
\Lambda_{\overline{\mathrm{MS}}}= & \frac{k}{3.334} \exp \left(\frac{-2 \pi}{\beta_{0} \alpha_{s}^{\mathrm{MOM}}}\right)\left[\left(\frac{\beta_{0} \alpha_{s} \mathrm{MOM}}{4 \pi}\right)^{2}\left(1+\frac{\beta_{1} \alpha_{s}{ }^{\mathrm{MOM}}}{2 \pi \beta_{0}}+\frac{\beta_{2} \alpha_{s} \mathrm{MOM}^{2}}{32 \pi^{2} \beta_{0}}\right)\right]^{\frac{\beta_{1}}{2 \beta_{0}^{2}}} \\
& \times \exp \left\{\frac{\beta_{0} \beta_{2}-4 \beta_{1}^{2}}{2 \beta_{0}^{2} \sqrt{\Delta}}\left[\arctan \left(\frac{\sqrt{\Delta}}{2 \beta_{1}+\beta_{2} \alpha_{s}{ }^{\mathrm{MOM}} / 4 \pi}\right)-\arctan \left(\frac{\sqrt{\Delta}}{2 \beta_{1}}\right)\right]\right\},
\end{aligned}
$$

where $\Delta \equiv 2 \beta_{0} \beta_{2}-4 \beta_{1}^{2}$, the unknown coefficient $\beta_{2}$ being fitted. The interplay between the ignorance of non-tree-level OPE correction and this fitted three-loop information is hoped to be controlled by studying the stability of the fits for different momentum windows.

The OPE approach used for propagator and symmetric three-point Green function is based on the factorization of the non-perturbative soft gluon contribution (related to the vacuum properties) and the perturbative hard gluons in the external legs of the Green functions. In the $\widetilde{\mathrm{MOM}}$ case, things are a little different. That has to be adapted since one of the external gluons of the Green function carries zero momentum. OPE large momentum expansion can 
nevertheless be done for the two other gluon fields carrying non-null momentum by following Eq. (1). As only an odd number of gluon field operators permits to build a Lorentz invariant v.e.v., non-null contributions will come now for terms of the Wilson expansion with an even number of local operators. Then, we will have

$$
\left\langle T\left(\widetilde{A}_{\mu}^{a}(-p) \widetilde{A}_{\nu}^{b}(p) \widetilde{A}_{\rho}^{c}(0)\right)\right\rangle=\left(\widetilde{c}_{1}\right)_{\mu \nu c^{\prime}}^{a b \rho^{\prime}}\left(p^{2}\right)\left\langle: A_{\rho^{\prime}}^{c^{\prime}}(0): \widetilde{A}_{\rho}^{c}(0)\right\rangle+\ldots
$$

As for the propagator or the symmetric vertex, it is immediate to prove that $\left(\widetilde{c}_{1}\right)_{\mu \nu c^{\prime}}^{a b \rho^{\prime}}\left(p^{2}\right)$ takes the tree-level expression for the asymmetric three-point Green function (with the explicit amputation of the zero-momentum gluon leg). Concerning subleading OPE corrections, they should be obtained from local operators of higher dimensions, and then a " $z o o$ " of different condensates appear. In principle, $\left\langle: A_{\mu^{\prime}}^{a^{\prime}} A_{\nu^{\prime}}^{b^{\prime}} A_{\rho^{\prime}}^{c^{\prime}}: \widetilde{A}_{\rho}^{c}\right\rangle,\left\langle: \partial_{\mu^{\prime}} \bar{c}^{a^{\prime}} c^{b^{\prime}}: \widetilde{A}_{\rho}^{c}\right\rangle$ or $\left\langle: \partial_{\rho^{\prime}} A_{\mu^{\prime}}^{a^{\prime}}(0) A_{\nu^{\prime}}^{b^{\prime}}(0): \widetilde{A}_{\rho}^{c}\right\rangle$, are all candidates to give non-null v.e.v. contributions. Furthermore, the problem due to the soft gluon in the vertex definition re-appears now in a new way: no v.e.v in the r.h.s of Eq. (19) involves directly the gluon condensate $\left\langle A^{2}\right\rangle$. Of course, this does not forbid an explanation of power corrections in terms of OPE for asymmetric $\alpha_{s} \widetilde{\mathrm{MOM}}$, but their non-perturbative condensates cannot be directly put in relation with those from symmetric $\alpha_{s}{ }^{\mathrm{MOM}}$ and gluon propagator. Consequently, with no additional phenomenological assumption no consistent test shall come from the running coupling constant in the asymmetric $\widehat{\mathrm{MOM}}$ scheme and it will be left aside in our present analysis.

\section{Fitting data to our ansätze}

Our goal is now to try a consistent description of lattice data for two- and three-point Green functions introduced in ref. [5-7] from the previously developed formulas for power corrections. Details of the lattice simulations, of the elaborated procedures to obtain an artifact-safe data set or of the definition of regularization-independent objects permitting lattice regularized data to be matched to continuum quantities in any scheme, can be found in those references. We directly show here the results of the matchings.

In a previous work [7], a fit of both the empiric coefficient for a power contribution and $\Lambda_{\overline{\mathrm{MS}}}$ were simultaneously performed by matching lattice data for $\alpha_{s} \widetilde{\mathrm{MOM}}\left(k^{2}\right)$ to a formula including non-perturbative additive power corrections. The $\Lambda_{\overline{\mathrm{MS}}}$ parameter thus obtained, $\Lambda_{\overline{\mathrm{MS}}}=237_{-13}^{+3}$ $\mathrm{MeV}$, is in total agreement with the one estimated by ALPHA group, $\Lambda_{\overline{\mathrm{MS}}}=238(19) \mathrm{MeV}$. The same kind of fit of $\alpha_{s} \widetilde{\mathrm{MOM}}\left(k^{2}\right)$ can be done by assuming the power correction to be proportional to $\alpha_{s} \widehat{\mathrm{MOM}}\left(k^{2}\right)$ analogously to what is done in the MOM case in eq. (17). The fitted $\Lambda_{\overline{\mathrm{MS}}}$ parameter raises in the latter fit to $\sim 250 \mathrm{MeV}$. This difference coming from the assumed $\propto \alpha_{s} \widetilde{\mathrm{MOM}}\left(k^{2}\right)$ dependence of the $1 / k^{2}$ term tells us something about a certain additional systematic uncertainty, not taken into account in ref. [7], due to neglecting anomalous dimensions of power corrections.

We will take the central value of the estimated $\Lambda_{\overline{\mathrm{MS}}}$ in ref. [7], $\Lambda_{\overline{\mathrm{MS}}}=237_{-13}^{+3} \mathrm{MeV}$, for $\alpha_{s}^{\mathrm{MOM}}$ and gluon propagator.

Two loop fits We have fitted the gluon propagator and the symmetric coupling constant according to eqs. (11, 12) and (17) with the leading perturbative parts computed to two loops. 
In both cases we fit the condensate. The result is:

$$
\text { propagator : } g_{R}^{2}\left\langle A^{2}\right\rangle_{R}=(2.32(6) \mathrm{GeV})^{2} ; \quad \alpha^{\mathrm{MOM}}: g_{R}^{2}\left\langle A^{2}\right\rangle_{R}=(4.36(12) \mathrm{GeV})^{2} .
$$

with $\chi^{2} /$ d.o.f $\simeq 1.2$. The clear disagreement between both estimates could be a sign that we are in the situation indicated in [12] that the perturbative order is too low to give an acceptable precision in the estimate of the power corrections. We need to check if going to three loops is enough.

Three loops fit $\beta_{2}^{\mathrm{MOM}}$ is not known. Then, our strategy will be to extract the value for the gluon condensate, $\left\langle A^{2}\right\rangle$, from the matching of (11,12) to gluon propagator lattice data; and then, by using that value, to search whether any sensible value of the unknown MOM coefficient $\beta_{2}$ allows to reproduce data for $\alpha_{s}{ }^{\mathrm{MOM}}(p)$.

In fig. 3 we plot the best fit of eq. (11, 12), for

$$
g_{R}^{2}\left\langle A^{2}\right\rangle_{R}=(2.76(4) \mathrm{GeV})^{2}
$$

to gluon propagator data. This error is computed by jacknife's method. The $\chi^{2}$ is 0.86 per d.o.f. in that fit, slightly better than for the two-loop fit. It is amusing that the three-loop condensate stemming from the propagator is not so different from the two-loop one.
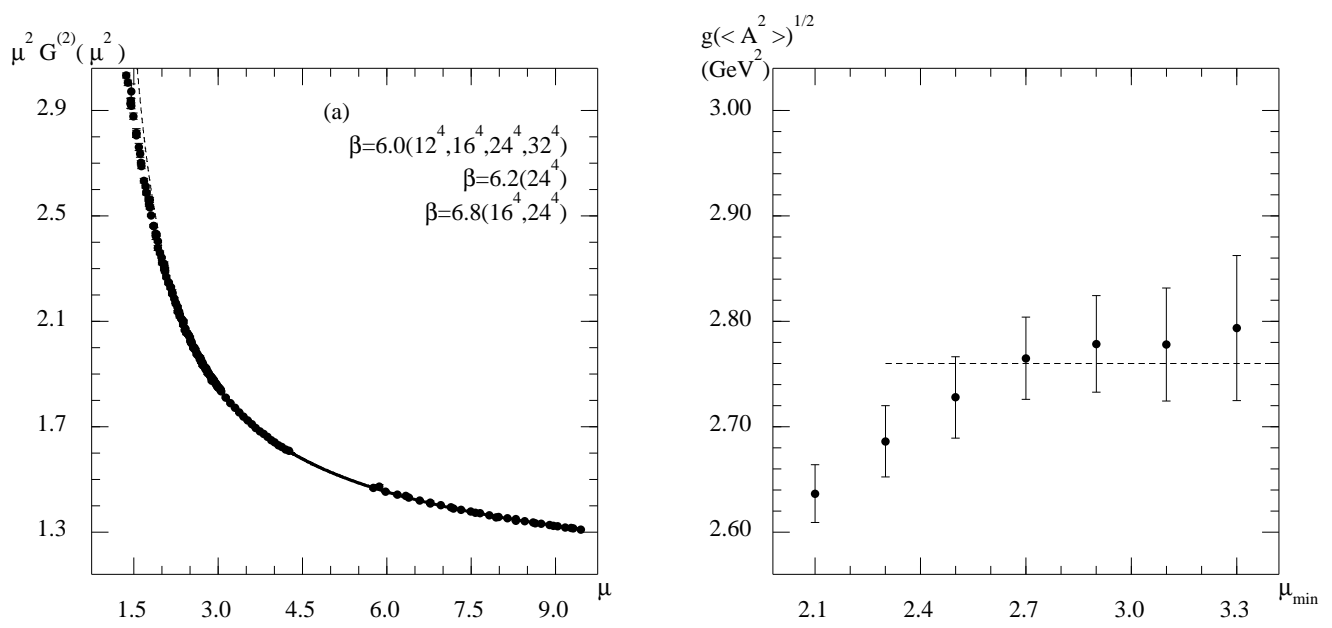

Figure 3: Formula (8), with the assumed value of $\Lambda_{\overline{\mathrm{MS}}}(237 \mathrm{MeV})$, fits impressively the data for $g^{2}\left\langle A^{2}\right\rangle=$ $(2.76(4) \mathrm{GeV})^{2}$ over a large momenta window from $2.6 \mathrm{GeV}$ to $\sim 10 \mathrm{GeV}$ (fig. a). The optimal window is fixed by requiring a reasonable stability of the fitted parameter $\left(g^{2}\left\langle A^{2}\right\rangle\right)$ for different choices of the lower momentum (fig. b).

With this value of the gluon condensate we find that

$$
\beta_{2}^{\mathrm{MOM}}=1.53(7) \times \beta_{2}^{\widetilde{\mathrm{MOM}}} \sim 7400 \pm 300
$$

optimises the quality matching $\left(\chi^{2} /\right.$ d.o.f. $\left.=1.1\right)$ of formula $(17)$ to lattice data (see fig. $\left.\mathbb{1}\right)$. 

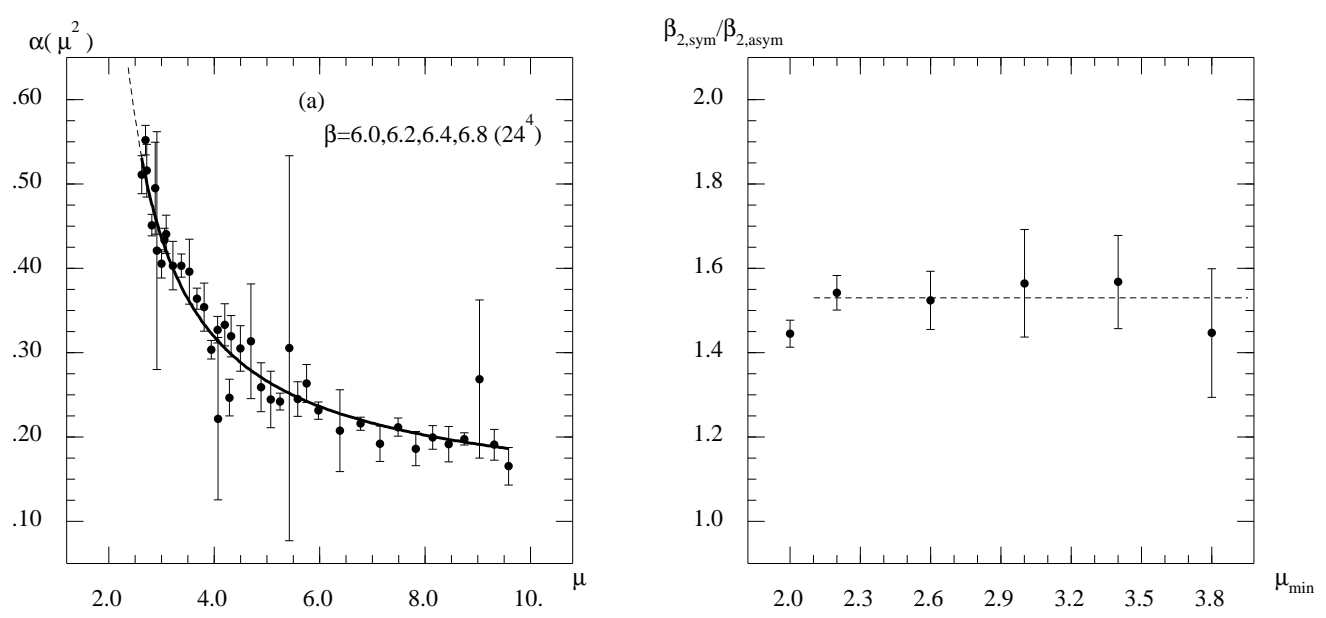

Figure 4: $\Lambda_{\overline{\mathrm{MS}}}$ assumed to be $237 \mathrm{MeV}$ and $g^{2}\left\langle A^{2}\right\rangle=0.236(7)$ from gluon propagator analysis, the best fit of Eq. $\sqrt{17}$ ) to lattice data (gives $\beta_{2}^{\mathrm{MOM}}=1.53 \times \beta_{2}^{\mathrm{MOM}}$ (fig. a). Fig. $b$ shows the stability of this parameter faced to different choices of the lower momentum of the fitting window.

\section{Discussion and conclusions}

A certain underestimation of the errors presented in the previous section should be admitted as a consequence of fixing $\Lambda_{\overline{\mathrm{MS}}}$ to its central value $237 \mathrm{MeV}$ of [7] without errors. We can correct for this by the same fitting strategy applied with different starting assumptions for $\Lambda_{\overline{\mathrm{MS}}}$, within its error interval. this results in a a wider dispersion:

$$
\beta_{2}^{\mathrm{MOM}} / \widehat{\beta_{2}^{\mathrm{MOM}}}=1.5(3)
$$

$\Lambda_{\overline{\mathrm{MS}}}$ and $\beta_{2}^{\mathrm{MOM}}$ appear to be strongly correlated. The goal of the present paper is less to give a reliable prediction of a certain perturbative parameter, as $\beta_{2}^{\mathrm{MOM}}$, than to carry out a preliminary study of the inner consistency from a description in terms of OPE predictions of lattice data. Let us repeat, the knowledge of three-loop information in the MOM scheme and the anomalous dimensions of the power corrections are prerequisite to any firm conclusion? The comparison of our estimate of $\beta_{2}^{\mathrm{MOM}}$ and the future perturbative computation of this parameter $\square$ are totally necessary to conclude whether signs of such a consistency are present or not.

Our analysis of lattice data from gluon propagator and running coupling constant unequivocally proves power corrections $\left(1 / p^{2}\right)$ to be necessary to match the data. The dimension 2 of power corrections clearly manifests the presence of non-gauge-invariant condensates (in particular, in our pure gauge QCD analysis, $A_{\mu} A^{\mu}$ ), if $\mathrm{OPE}$ is invoked to explain the results. Since we work in the Landau gauge the appearance of such a non-gauge-invariant condensate is in full agreement with all theoretical expectations. The value for $\sqrt{g_{R}<A^{2}>_{R}}$ around $3 \mathrm{GeV}$ might seem unexpectedly large but this is a subjective matter. The perturbative Wilson coefficients

\footnotetext{
${ }^{9} \mathrm{~A}$ very recent lattice calculation within the two-dimensional non-linear sigma model $[16]$ insists on the necessity of knowing the OPE coefficients at least to one loop to reach agreement with OPE predictions.

${ }^{10} \mathrm{~A}$ computation of $\beta_{2}$ may appear soon $[17$.
} 
for the OPE of the involved Green functions have been theoretically computed. Then, in order to satisfy the universality of the non-perturbative gluon condensate appearing directly in OPE of both gluon propagator and MOM coupling constant, we need to assume $\beta_{2}^{\mathrm{MOM}}=7400(1500)$. The perturbative computation of this parameter as well as that of the anomalous dimensions of the power corrections will either exclude the interpretation of power corrections just in terms of universal gluon condensates at three loops, or on the contrary throw a positive sign supporting the usual "QCD sum rules" procedure to connect non-perturbative effects for different correlation functions. All the theoretical tools for such a OPE testing procedure have been developed in the present paper.

\section{Acknowledgments}

We specially thank D. Becirevic for thorough discussions at the earlier stages of the work. We are also indebted to Y. Dokshitzer and G. Korchemsky for several inspiring comments. J. R-Q is indebted to Spanish Fundación Ramón Areces for financial support. These calculations were performed on the QUADRICS QH1 located in the Centre de Ressources Informatiques (Parissud, Orsay) and purchased thanks to a funding from the Ministère de l'Education Nationale and the CNRS.

\section{References}

[1] S. Capitani, M. Guagnelli, M. Lüscher, S. Sint, R. Sommer, P. Weisz and H. Wittig, Nucl. Phys. Proc. Suppl. 63 (1998) 153; Nucl. Phys. B544 (1999) 669.

[2] G.S. Bali and K. Schilling, Phys. Rev. D47 (1993) 661.

[3] G.P. Lepage and P. Mackenzie, Phys. Rev. D48 (1992) 2250; G. de Divitiis et al., Nucl. Phys. B433 (1995) 390; Nucl. Phys. B437 (1995) 447.

[4] B. Allés, D. S. Henty, H. Panagopoulos, C. Parrinello, C. Pittori, D. G. Richards, Nucl. Phys. B502 (1997) 325.

[5] Ph. Boucaud, J. P. Leroy, J. Micheli, O. Pene, C. Roiesnel, J. High Energy Phys. 10 (1998) 017; J. High Energy Phys. 12 (1998) 004.

[6] D. Becirevic, Ph. Boucaud, J. P. Leroy, J. Micheli, O. Pene, J. Rodriguez-Quintero, C. Roiesnel, Phys. Rev. D60 (1999) 094509; Phys. Rev. D61 (2000) 114508.

[7] Ph. Boucaud et al., J. High Energy Phys. 04 (2000) 006.

[8] G Burgio, F. Di Renzo, G. Marchesini and E. Onofri, Phys. Lett. B422 (1998) 219; G. Burgio, F. Di Renzo, C. Parrinello and C. Pittori Nucl. Phys. Proc. Suppl. 73 (1999) 623; Nucl. Phys. Proc. Suppl. 74 (1999) 388.

For reviews and classic references see:

V.I. Zakharov, Nucl. Phys. B385 (19452) 1992;

A.H. Mueller, in QCD 20 years later, vol. 1 (World Scientific, Singapore 1993). B. Lautrup, Phys. Lett. B69 (1977) 109; G. Parisi, Phys. Lett. B76 (1977) 65; Nucl. Phys. B150 (1979) 163; G. t'Hooft, in The Whys of Subnuclear Physics, Erice 1977, ed A. Zichichi, (Plenum, New York 1977); M. Beneke and V.I. Zakharov, Phys. Lett. B312 (1993) 340; M. Beneke Nucl. Phys. B307 (1993) 154; A. H. Mueller, Nucl. Phys. B250 (1985) 327; Phys. Lett. B308 (1993) 355; G. Grunberg, Phys. Lett. B304 (1993) 183; Phys. Lett. B325 (1994) 441. 
[9] M. Lavelle and M. Oleszczuk, Mod. Phys. Lett. A 7 (1991)3617; J. Ahlbach, M. Lavelle, M. Schaden, A. Streibl, Phys. Lett. B275 (1992) 124.

[10] M.A. Shifman, A.I. Vainshtein, V.I. Zakharov, Nucl. Phys. B147 (1979) 385,447,519; M.A. Shifman, A.I. Vainshtein, M.B. Voloshin, V.I. Zakharov, Phys. Lett. B77 (1978) 80; S. Weinberg, The quantum theory of fields, vol. 2 (Cambridge University Press 1996)

[11] F. David, Nucl. Phys. B234 (1984) 237.

[12] G. Martinelli, C. Sachrajda, Nucl. Phys. B478 (1996) 660.

[13] A.I. Davydychev, P. Osland, O.V. Tarasov, Phys. Rev. D58 (1998) 036007; S.A. Larin, J.A.M. Vermaseren, Phys. Lett. B303 (1993) 334.

[14] K. Chetyrkin, A. Rétey, hep-ph/0007088.

[15] Ph. Boucaud, A. Le Yaouanc, J.P. Leroy, J. Micheli, O. Pène, J. Rodríguez-Quintero, work in progress.

[16] S.Caracciolo, A. Montanari and A. Pelissetto, hep-lat/0007044.

[17] K. Chetyrkin, private communication. 